\title{
First detection of a human astrovirus type 8 in a child with diarrhea in Belém, Brazil: comparison with other strains worldwide and identification of possible three lineages
}

\author{
Yvone B Gabbay $/^{+}$, Alexandre C Linhares, Darleise S Oliveira, Lilian S Nakamura, \\ Joana D'Arc P Mascarenhas, Rosa Helena P Gusmão*, Marcos B Heinemann**, \\ Olinda Macêdo, José Paulo G Leite***
}

\begin{abstract}
Seção de Virologia, Instituto Evandro Chagas, Secretaria de Vigilância em Saúde, Ministério da Saúde, BR 316 Km 7, s/nº, 67030-000 Ananindeua, PA, Brasil *Universidade Estadual do Pará, Belém, PA, Brasil **Escola de Veterinária, Universidade Federal de Minas Gerais, Belo Horizonte, MG, Brasil ***Departmento de Virologia, Instituto Oswaldo Cruz-Fiocruz, Rio de Janeiro, RJ, Brasil
\end{abstract}

This study describes the genetic relationships of the first human astrovirus type-8 (HAstV-8) detected in Belém-Brazil, during a public hospital-based study. This strain was compared with other HAstV-8 strains identified elsewhere which have sequences available at GeneBank. The regions ORF1a (primers Mon348/Mon340) and ORF2 (primers Mon269/Mon270) were analyzed by nucleotide sequencing and a high similarity rate was observed among the Belém strain and other HAstV-8 strains. In ORF1a, homology values of 93-100\% were detected, and in ORF2 96-99\%. Considering the sequence variation (7\%) observed in ORF2 region, it was suggested that HAstV-8 strains could be divided in three different lineages.

Key words: astrovirus type 8 - molecular characterization - Belém - Brazil

Human astrovirus (HAstVs) belongs to the Astroviridae family, and are classified in the genus Mamastrovirus (Monroe et al. 2005). They are small, round non-enveloped viruses, $28 \mathrm{~nm}$ in diameter and composed of a single-stranded positive-sense RNA genome (ssRNA). The genome contains three open reading frames (ORFs) designated ORF1a, ORF1b, and ORF2, as well as terminal non-coding regions (NCR). ORF1a and $1 \mathrm{~b}$ encode non-structural proteins, including a serine protease (Pro) and RNA-dependent RNA polymerase $(\mathrm{Pol})$, respectively, and these regions contain highly conserved sequences. ORF2 is located at the 3 ' end of the genome, encodes the capsid protein precursor, and is highly variable (Matsui \& Greenberg 2001, Walter et al. 2001).

HAstVs were classified into eight genotypes based on the nucleotide sequence analysis of a 348-bp region of ORF2 (Mustafa et al. 2000). These eight genotypes showed good correlation with the eight serotypes determined according to the reactivity of the capsid proteins with type-specific monoclonal antibodies (Sakamoto et al. 2000, Taylor et al. 2001).

For the eight types of HAstV already reported, the HAstV- 1 is considered the most prevalent and HAstV-6, 7, and 8 as the seldom detected (Mustafa et al. 2000, Guix et al. 2002, Méndez-Toss et al. 2004). After the description of the prototype HAstV-8 (Z66541) in United Kingdom in 1995, the first reports describing the detection of this type of virus relate to the occurrence of

Financial support: IEC/SVS/MS, IOC-Fiocruz, SECTAM/Funtec/ $\mathrm{PA}, \mathrm{Pibic} / \mathrm{CNPq}$

${ }^{+}$Corresponding author: yvonegabbay@iec.pa.gov.br

LSN is CNPq fellowship

Received 16 January 2007

Accepted 22 March 2007 single isolates like those occurring in Ghaza and Uganda (Monceyron et al. 1997), Pakistan (Sakamoto et al. 2000), Australia (Nadan et al. 2003) and, more recently, in the United States (acces. nos AY304469 and AY304470), Brazil (Silva et al. 2006, and acces. nos DQ381506 to DQ381508), and India (acces. nos $\mathrm{AB} 116554, \mathrm{AB} 126670$ to 126674, AB191789, AB194280, and AB211059). Studies conducted in Egypt, Spain, Korea, South Africa, Hungary, and Mexico demonstrated the presence of HAst $\mathrm{V}-8$ in percentages that varied from 8 to $23 \%$, considering the total of positive cases detected (Naficy et al. 2000, Guix et al. 2002, Kang et al. 2002, Nadan et al. 2003, Méndez-Toss et al. 2004, Jakab et al. 2004).

One strain of HAstV-8 (PA-NSC087-BR) was detected in Belém, North of Brazil, during a study conducted in a public hospital from November 1992 to November 1994 involving children aged 0-5 years, who were admitted with acute gastroenteritis (Gusmão et al. 1999). Such HAtV-8 isolate was obtained from a seven-month old female with acute diarrhea of four-day duration, which persisted for eight more days. Moderate dehydration was identified during the first $48 \mathrm{~h}$ of hospitalization. In addition, fever was recorded in the first day and acute respiratory infection was diagnosed on the second and third days of admission. A diarrheic stool sample was obtained four days after admission (March 1st, 1993), being stored at Instituto Evandro Chagas, at $-20^{\circ} \mathrm{C}$, until being processed.

This was the first time that a HAstV-8 was detected in Belém. Furthermore, this type has rarely been detected worldwide (Guix et al. 2002, Méndez-Toss et al. 2004), and up to now, only two studies described molecular analysis of the HAstV-8 strains (Méndez-Toss et al. 2000, Taylor et al. 2001). The aim of this report is therefore to describe the antigenic and genetic relationships of this HAstV-8 strain (PA-NSC087-BR), in light of compari- 
son with other HAstV-8 strains identified elsewhere, to gathering more complete characterization data on this type. This is the first approach to compare different strains of HAstV-8 already described in relation to the ORF1a and 5' end ORF2 region of the HAstV-8.

The specimens collected in this study were screened for the presence of HAstV antigen by a commercial qualitative enzyme immunoassay (EIA) kit (IDEIA ${ }^{\mathrm{TM}}$ Dako Cytomation, Ely, UK), with subsequent confirmation by reverse transcription-polymerase chain reaction (RTPCR) using specific primers Mon 269 (4526-4545 nt) and Mon 270 (4955-4974 nt), yielding an expected product size of $449 \mathrm{nt}$ in the 5' end of ORF2, as described previously by Noel et al. (1995) with few modifications (Gabbay et al. 2005). The positive samples by RT-PCR were genotyped by two methodologies: the type-specific RT-PCR using primers described by Sakamoto et al. (2000), that amplified the 3 ' end region of ORF2, and by the nucleotide sequencing of RT-PCR astrovirus amplicons that was carried out under the conditions described by Gabbay et al. (2005), with the primers Mon 269 / Mon 270. Another pair of primers (Mon 348 / Mon 340), directed towards the ORF1a (Belliot et al. 1997), was also used with the HAstV-8 sample, to obtain data from another region of the genome, therefore broadening the phylogenetic analysis. Sequence data obtained with sample PA-NSC087-BR with primers Mon 269 / Mon 270 were aligned and edited using BioEdit Sequence Alignment Editor (v.7.0.5.2) program and compared with the eight prototype sequences (HAstV1 [L23513], HAstV2 [L13745], HAstV3 [L38505], HAstV4 [L38506], HAstV5 [U15136], HAstV6 [L38507] and HAstV7 [L38508], Oxford reference strains and HAstV-8 [Z66541], United Kingdom reference) and with the strains Yuc-8 (AF260508), AS20 (AF292079), Melb8A (AF175261), RJ8398/BR/04 (DQ381508), Bcn8-5 (AF34880), BrG8 (DQ139832) and RJ8163/ BR/04 (DQ381507) obtained from GenBank (www.ncbi.nlm.nih.gov), using the BLAST program. The sequence data obtained with sample PA-NSC087-BR with primers Mon 340 / Mon 348 were aligned with the sequences AS20 (AF290509), KS106205 (AF361030), KS 106207 (AF361032), HAstV-2 (L13745), BrG5 (DQ028633), HAstV-3 (AF141381), HAstV-1 (L23513), BrG4 (DQ070852), V770/2002 (AB126670), I/V813/ 2002 (AB126672), Yuc-8 (AF260508), HAstV-6 (AF290507), HAstV-7 (AF290508). Phylogenetic trees were constructed by neighbor-joining method using the Mega (v. 3.1) software, supported by bootstrap using 2000 replicates. The nucleotide sequences determined in this study have been deposited in the GenBank database and assigned the accession numbers DQ990460 and DQ990461.

All the specimens of this study were tested for the presence of bacteria and parasites following the specifications of the "WHO Manual for Laboratory Investigation of Acute Enteric Infections, Programme for Control of Diarrhoeal Diseases". Samples were also tested for rotavirus antigen using a commercial EIA (DAKOPATTS EIA kits-Copenhagen, Denmark), and for calicivirus using the RT-PCR technique including a pair of primers p289 and p290 as described by Jiang et al. (1992).

After the detection of HAstV by both EIA and RT-
PCR, the sample PA-NSC087-BR was tested by the typespecific PCR that assigned this sample as a HAstV-8. Nucleotide sequence analyses using the pair of primers Mon 269/Mon 270 and Mon 340/Mon 348 confirmed this strain as belonging to genotype 8. A good correlation between these two methodologies was also demonstrated in studies conducted in a periurban community of Mexico City and Hungary for all genotypes (Walter et al. 2001, Jakab et al. 2004).

Phylogenetic trees based on the 348-bp fragment of the ORF2 region (Fig. 1A) and on the 246-bp fragment of the ORF1a region (Fig. 1B) were constructed to assess the genetic relatedness between the PA-NSC087BR strain and other available sequences of HAsTV-8 obtained from different countries.

Analysis of the ORF1a region showed that PA-NS C087-BR group together with the other HAstV-8 genotypes as well as with genotypes HAstV-1 to HAstV-5 in a genogroup A, as defined by Belliot et al. (1997) and Taylor et al. (2001). Comparing the strain PA-NSC087-BR with the strain Yuc-8, a 97\% nt and a 100\% aa similarities were observed. With the other HAstV-8 (AS20, KS106205, KS106207, and V813/2002) the similarities were $93-94 \%$ at nt level and 96-99\% at aa level. In one HAstV-8 sample from India (V770/2000), the sequence described in GeneBank for ORF1a region was a little small (220 bp), and when it was compared with the strain PA-NSC087-BR a similarity of $93 \%$ in nt and of $98 \%$ in aa was seen. The comparison of the strain PANSC087-BR with the strain classified as HAstV-1, 2, 3, 4 , and 5 showed significant homology (90-93\% in nt and 96-97\% in aa), in contrast with HAstV types 6 and 7 where similarity was lower (80\% nt and $92-95 \%$ aa).

As previously demonstrated (Belliot et al. 1997, Taylor et al. 2001), the nucleotide sequence analysis of ORF1a region from HAstV-1 to HAstV-8 indicate that HAstVs fall into two distinct genogroups. This fact was also observed in this study however, we did not verify a high similarity between HAstV-8 and HAstV-4 strains, as reported by Taylor et al. (2001). This may be explained by the fact that HAstV-4 strain used in our analysis was different from that utilized in that research.

Considering the amino acid sequence of the ORF1a region, the strain PA-NSC087-BR demonstrated a pattern similar to that of strain Yuc-8, and a difference of one (V770/2002 and V834/2000), two (AS20 and KS106207), and three (KS106205) amino acids as compared to other strains sequenced (Fig. 2A).

Analysis of the ORF2 region indicates that PANSC087-BR strain has a higher pairwise similarity with the strain SXPAKAS706 (99\% in nt), and with the other strains (Yuc-8, AS20, RJ8398, Bcn8-5, BrG8, Melb8A), of $96-98 \%$ in nt. A divergence of $6 \%$ in nt sequence was noted in relation to the HAstV-8 prototype, and of 7\% with the HAstV-8 strain RJ8163. Based on the sequence variations observed among strains of a same type, some authors (Medina et al. 2000, Guix et al. 2002) have proposed that strains showing a sequence diversity of at least $7 \%$ could be classified as new lineages. Using this same criterion, we observed, as for the ORF2 region, HAstV8 could be divided in three different lineages and two of these were detected in Brazil (Fig. 1A). 

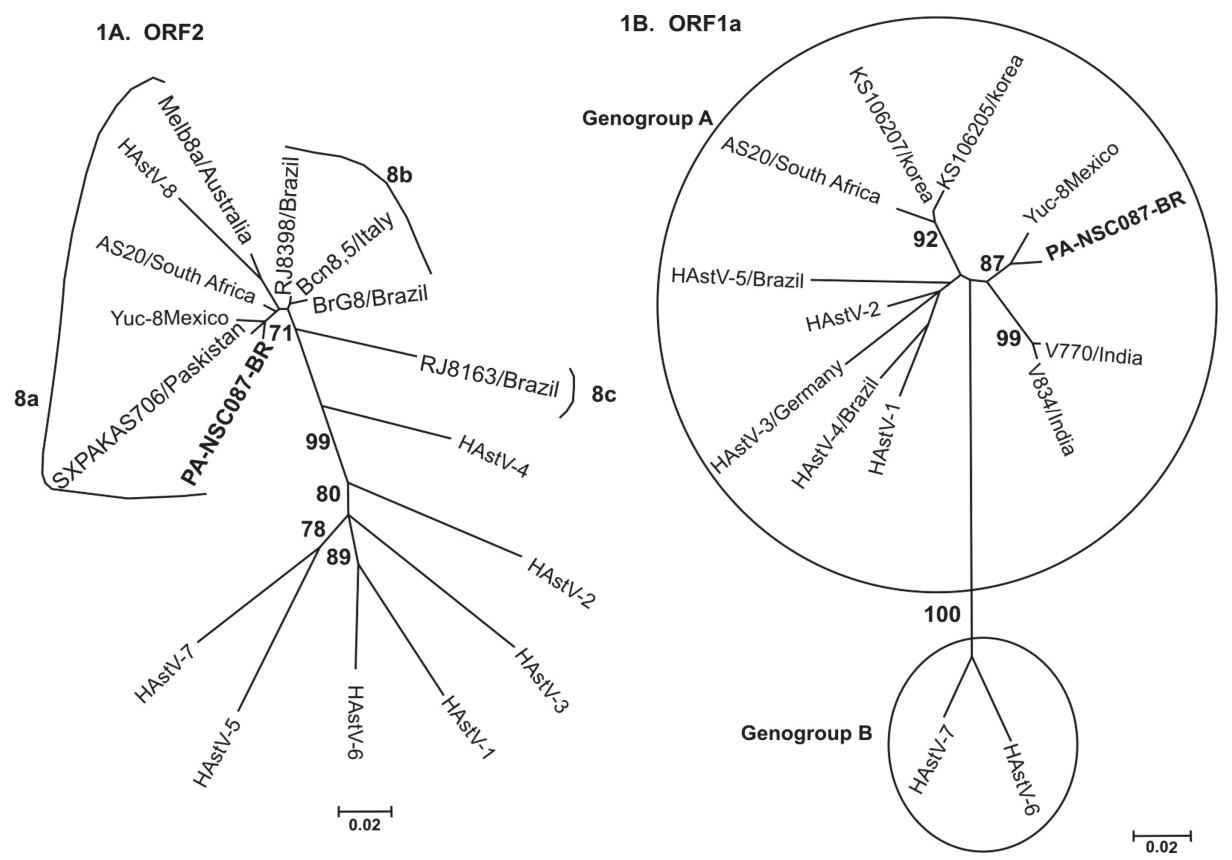

Fig. 1: neighbor-joining unrooted phylogenetic trees of a 348-bp ORF2(A) and 246-bp fragment of the ORF1a(B) regions of human astrovirus (HAstV) strains, including the strain PA-NSC087-BR from Belém, Brazil, the standard HAstV strains (HAstV-1 to HAstV-8) and HAstV-8 strains from database. Country of origin/data of the strains was shown in the tree.

A. ORF1a

Yuc-8

PA-NSC087-BR

AS20

KS106207

KS106205

$\mathrm{V} 770 / 2002$

v834/2002

B. ORF2

Oxford
PA-NSC087-BR
PAKAS706
Yuc-8
AS20
Melb8A
RJK8398
Ben8.5
BrG8
RJ8163

NT

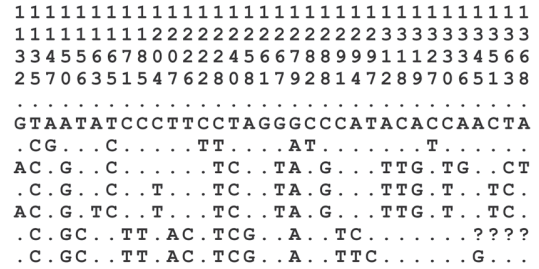

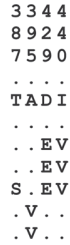

NT

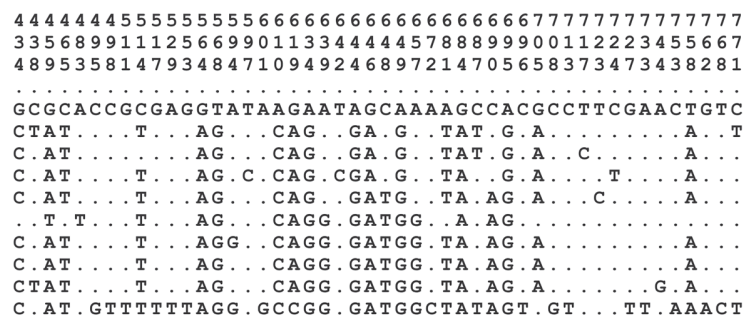

AA

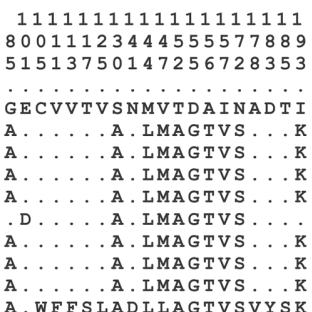

Fig. 2: differences observed during the alignment of nucleotide and amino acid sequences A: 246-bp fragment of the ORF1a region of human astrovirus gene sequence of PA-NSC087-BR and more five HAstV-8 strains, in comparison with the strain Yuc-8 from Mexico; B: 348-bp ORF2 region of human astrovirus gene sequence of PA-NSC087-BR and more eight HAstV-8 strains, in comparison with the HAstV-8 Oxford prototype. The number in the top demonstrated the position of the nucleotide/amino acid in the sequence. A dot indicates nucleotide/amino acid identity with the comparison strain sequence at that position.

In relation to the amino acid sequence, a similarity of $100 \%$ was noted in the ORF2 region, when comparing the PA-NSC087-BR strain with the other HAstV-8 strains (Pakistan, Mexico, South Africa, Brazil, and Spain), except for Melb8A (97\%) and RJ8163 (91\%) strains. When comparing to the HAstV-8 prototype, the similarity was of $91 \%$. The comparison between PANSC087-BR strain and HAstV-1 to 7 denotes a closer relationship with HAstV-4 (similarity of 90\% nt and 99\% aa) than with the other types (similarity 78-82\% nt and 91$96 \%$ aa). This is in agreement with observations made by Taylor et al. (2001) during the characterization of the strain AS20 from South Africa, and by Mendéz-Toss et al. (2000) during an analysis of Yuc-8 strain from Mexico.

Comparing the fragment of 348-bp of the ORF2 region obtained from the HAstV-8 prototype with those from PA-NSC087-BR, PAKAS706, Yuc-8, AS20, RJ $8398, \mathrm{Bcn} 8.5$, and BrG8 strain, a difference of 10 amino 
acids was observed (Fig. 2B). The strains Melb8A and RJ8163 showed a difference to the prototype of 9 and 19 amino acids, respectively. This clearly demonstrated the great difference between the prototype and the other type 8 strains circulating worldwide and sustains the classification of this type into three lineages. The significant correlation (97 to 99\%) observed between the strain detected in Belém and other from various countries $(\mathrm{Pa}-$ kistan, Mexico, South Africa, Spain, and Brazil), suggests that a genetically similar HAstV-8 strain was circulating in these several countries.

No other enteropathogen was detected in the PANSC087-BR sample, suggesting that astrovirus had a role in the etiology of diarrhea. Of note, the clinical course appeared to be more severe than it has previously been appreciated (Nadan et al. 2003).

We cannot rule out the possibility of a nosocomial infection since the fecal sample of this child was obtained four days after admission; however, there is a strong evidence of community-acquired infection because we had a single HAstV-8 detection into the ward environment. It is important to mention that during this study a total of 372 specimens were tested yielding an astrovirus positivity of $8.9 \%$ (33/372), and with the detection of three HAstV-1 nosocomial infections.

In summary, we characterized the first case of HAst V-8 isolate in Belém, Brazil, emphasizing that comparison of sequences obtained from the ORF2 region allowed us to sustain the existence of three distinct lineages within HAstV-8.

\section{ACKNOWLEDGMENTS}

To the valuable technical support provided by Maria Silvia de Lucena.

\section{REFERENCES}

Belliot G, Laveran H, Monroe SS 1997. Detection and genetic differentiation of human astroviruses: phylogenetic grouping varies by coding region. Arch Virol 142: 1323-1334.

Gabbay YB, Luz CRNE, Costa IV, Cavalcante-Pepino EL, Sousa MS, Oliveira KK, Wanzeller ALM, Mascarenhas JDP, Leite JPG, Linhares AC 2005. Prevalence and genetic diversity of astrovirus in children with and without diarrhea in São Luís, Maranhão, Brazil. Mem Inst Oswaldo Cruz 100: 709-714.

Guix S, Caballero S, Villena C, Bartolome R, Latorre C, Rabella N, Simo M, Bosch A, Pinto M 2002. Molecular epidemiology of astrovirus infection in Barcelona, Spain. J Clin Microbiol 40: 133-139.

Gusmão RHP, Mascarenhas JDP, Gabbay YB, Lins-Laison ZC, Ramos FLP, Monteiro TAF, Valente SA, Fagundes-Neto U, Linhares AC 1999. Rotavirus subgroups, G serotypes, and electrophoretypes in cases of nosocomial infantile diarrhoea in Belém, Brazil. J Trop Pediat 45: 81-86.

Jakab F, Meleg E, Bányai K, Melegh B, Tímár L, Péterfai J, Szücs G 2004. One-year survey of astrovirus infection in children with gastroenteritis in a large hospital in Hungary: occurrence and genetic analysis of astroviruses. J Med Virol 74: 71-77.

Jiang X, Wang J, Graham DY, Estes MK 1992. Detection of Norwalk virus in stool by polimerase chain reaction. $J$ Clin Microbiol 30: 2529-2534.

Kang Y-H, Park Y-K, Ahn J-B, Yeun J-D, Jee Y-M 2002. Iden- tification of human astrovirus infections from stool samples with diarrhea in Korea. Arch Virol 147: 1821-1827.

Matsui SM, Greenberg HB 2001. Astroviruses. In DM Knipe, PM Howley (eds), Fields Virology, 4th ed., Lippincott, Williams and Wilkins, Philadelphia, p. 875-893.

Medina SM, Guitierrez MF, Liprandi F, Ludert JE 2000. Identification and type distribution of astroviruses among children with gastroenteritis in Colombia and Venezuela. J Clin Microbiol 38: 3481-3483.

Méndez-Toss M, Griffin DD, Calva J, Contreras JF, Puerto FI, Mota F, Guiscafre H, Cedillo R, Munoz O, Herrera I, Lopez S, Arias CF 2004. Prevalence and genetic diversity of human astroviruses in Mexican children with symptomatic and asymptomatic infections. J Clin Microbiol 42: 151-157.

Méndez-Toss M, Romero-Guido P, Munguía ME, Méndez E 2000. Molecular analysis of a serotype 8 human astrovírus genoma. J Gen Virol 81: 2891-2897.

Monceyron C, Grinde B, Jonassen TØ 1997. Molecular characterization of the 3'-end of the astrovirus genome. Arch Virol 142: 699-706.

Monroe SS, Carter MJ, Herrmann JE, Mitchell DK, SanchezFauquier A 2005. Family Astroviridae. In CM Fauquet, MA Mayo, J Maniloff, U Desselberger, LA Ball (eds), Virus Taxonomy: Eighth Report of the International Committee on Taxonomy of Viruses, Elsevier/Academic Press, London, p. 859-864.

Mustafa H, Palombo EA, Bishop RF 2000. Epidemiology of astrovirus infection in young children hospitalized with acute gastroenteritis in Melbourne, Australia, over a period of four consecutive years, 1995 to 1998. J Clin Microbiol 38: 1058-1062.

Nadan S, Walter JE, Grabow WOK, Mitchell DK, Taylor MB 2003. Molecular characterization of astroviruses by reverse transcriptase PCR and sequence analysis: comparison of clinical and environmental isolates from South Africa. Appl Environ Microbiol 69: 747-753.

Naficy AB, Rao MR, Holmes JL, Abu-Elyazeed R, Savarino SJ, Wierzba TF, Frenck RW, Monroe SS, Glass RI, Clements JD 2000. Astrovirus diarrhea in Egyptian. J Infect Dis 182: 685-690.

Noel JS, Lee TW, Kurtz JB, Glass RI, Monroe SS 1995. Typing of human astroviruses from clinical isolates by enzyme immunoassay and nucleotide sequencing. J Clin Microbiol 33: 797-801.

Sakamoto T, Negidhi H, Wang QH, Akihara S, Kim B, Nishimura S, Kaneshi K, Nakaya S, Ueda Y, Sugita K, Motohiro T, Nishimura T, Ushijima H 2000. Molecular epidemiology of astroviruses in Japan from 1995 to 1998 by reverse transcription-polymerase chain reaction with serotype-specific primers (1 to 8). J Med Virol 61: 326-331.

Silva PA, Cardoso DDP, Schreier E 2006. Molecular characterization of human astroviruses isolated in Brazil, including the complete sequences of astrovirus genotypes 4 and 5 . Arch Virol 151: 1405-1417.

Taylor MB, Walter J, Berke T, Cubitt WD, Michell DK, Matson DO 2001. Characterization of a South African human astrovirus as type 8 by antigenic and genetic analyses. $J$ Med Virol 64: 256-261.

Walter JE, Mitchell DK, Guerreiro ML, Berke T, Madison DO, Monroe SS, Pickering LK, Ruiz-Palacios G 2001. Molecular epidemiology of human astrovirus diarrhea among children from a periurban community of Mexico city. J Infec Dis 183: 681-683. 